\title{
People as Sensors for Smart City
}

\author{
${ }^{1}$ Sagar Vaidya ${ }^{2}$ Prathamesh Shingvi ${ }^{3}$ Kamalesh Wankhede ${ }^{4}$ Akshay Sharma \\ Department of Computer Engineering, \\ Sinhgad College of Engineering, \\ Vadgaon, Pune-411041
}

\begin{abstract}
A city becomes smart when common problems like traffic and issues related to local governance are successfully dealt with. Today the biggest problem any city faces is the increase in traffic due to increasing population of people and vehicles. Real time data obtained from smartphones as well as data collected over a significant period of time can help us in solving this problem to some extent. A system is proposed which uses people as sensors for solving the common problem of any city which is traffic. People's participation can help in bringing effective changes in local governance. A communication network that spans across an entire city will be built so that people can contribute to the betterment of themselves. The application under consideration is assumed to be built for the local governing body. Hence officials from various departments of the governing body can have direct communication with the common man of a city. This can indirectly prevent malpractices like corruption. Any end user will be able to raise his voice against any grievance he faces which on approval by the system and if well supported by other users, will be notified to the concerned authority with the help of notifications.
\end{abstract}

\section{General Terms}

Internet of things, Web services

\section{Keywords}

Global Positioning System, Google API's, Android Smartphones, Shortest path

\section{INTRODUCTION}

In today's world, Information and Communication Technologies play a crucial role in making cities smarter [1], more accessible and more open. Today real time data is of utmost importance. Majority of people possess a smartphone. The proposed application aims to make use of this real time data from people themselves, for navigating to the required location in shortest time. People's participation can bring about effective changes in local governance of a city. One of the important aspects of making a city smarter is by improving its traffic management. It is seen that traffic scenarios at certain areas in city has a pattern associated with it. Roads in the proximity of industrial areas have more traffic during start or end of shifts in companies. Roads near schools are flooded with vehicles during start and end of school hours. The user will be able to feed traffic information of nearby roads provided with the help of his GPS location. The users which want to travel from their source to destination can use this information by showing them the shortest path available from the alternatives. It would be constantly running on the user's device during his journey to provide real-time data so that the path will re-route if there are traffic variations in the previous path. Google map API's are used for the design of the map whereas the database obtained from users along with Google direction API's,the shortest path is computed. Studying these patterns, a novel solution is provided to overcome the traffic chaos in urban areas.

\section{RELATED WORK}

2.1 Hart proposed A*algorithm It is based on a greedy best-first search algorithm. $\mathrm{A}^{*}$ algorithm is used in path finding and for graph traversals. It uses a heuristic function for the evaluation while calculating path from source to destination. The heuristic function is later divided into two functions: the path-cost function and the heuristic estimate function[2]. The path-cost function is the total cost from source location to current location while the heuristic estimate function is used in the estimation of cost from current location to destination location. It is also used for optimal parking path[3].

\subsubsection{Limitation}

Time consuming.

\subsection{Halvey proposed the average time segmentation method}

It uses the average values which increase and decrease a userspecified range as the time segmenting positions. However, the user-specified range is still a difficult definition value [4].

\subsubsection{Limitation \\ Less accurate.}

\subsection{Gonzalez proposed method to calculate shortest path}

It uses environmental factors that may reflect various influences of the velocity of vehicle, and then these factors are used to build the decision tree. The current environmental situations are used to evaluate the velocity of the vehicle[5].

\subsubsection{Limitation}

Not accurate due to environmental factor.

\subsection{Wang Wei proposed an alarm notification for traffic accidents}

It can automatically detect a traffic accident, search for the spot and then helps to send the required information to nearby first aid center within a few seconds covering geographical coordinates, the different time and circumstances in which any traffic related accident takes place[6]. With the help of satellite navigation system it helps the nearby first aid rescuers to reach the location.

\subsubsection{Limitation}

Additional hardware required and hence costly then using an Android device.

\section{PROJECT OBJECTIVES}

\subsection{Primary Objectives}

The main objective of the project is to use real time data from people and use it for their own benefit. Solving traffic problems of a city is the prime focus of the project.

The System includes the following features: 


\subsubsection{Traffic Detection}

\subsubsection{Alert Generation}

\subsubsection{Notifying traffic scenario to registered users \\ 3.1.4 Suggestion of alternative path}

\subsection{Secondary Objectives}

Italso provides a platform where users can post their issues regarding local governance which can be notified to concerned authority. The grievances posted by the users may be accidents at specific locations or even potholes and roads which require maintenance. The users can send the location, information about the issue along with the image of the issue. As more and more people start rating this grievance, it will be sent to the concerned authority for the immediate maintenance based on its priority.

\section{OPERATIONAL DETAILS \\ 4.1 Android Development Tools}

Android Development Tools (ADT) is a plugin for the Eclipse IDE which is used to create the graphical user interface for the client side, as well as the queries which are going to be sent to the server. All the different layouts and the class files are created using the ADT. Different layouts are created like user registration form to create a new user for the application, login page for logging existing users, a layout for selecting the map for travelling from source to destination and the other choice to enter the traffic at a particular location. It also has a layout to post grievances with forms on describing the issue along with images showing the particular issue.

Android Development Tools helps in fast development of android applications along with connectivity with the server and it also helps in publishing the application on the android market with the signed version of the apk. Many different guides and video tutorials are present to help in the development of applications in ADT.

\section{2 .NET Framework}

.NET Framework is a software framework developed by Microsoft. This framework along with $\mathrm{C \#}$ is used for server side coding, while a dedicated server is used to deploy the project data to this server. Microsoft Visual Studio is an integrated development environment (IDE) form Microsoft. It is used to create the database for server side along with shortest distance calculation by comparing the data in the database with the actual data provided by Google API's.

\subsection{Google Maps API}

With the Google Maps Android API, maps can be easily added to the application based on Google Maps data. Google Maps servers, data downloading, map display, and response to map gestures are easily handled using the Google Maps API. The Google Maps API helps us to show a complete map for users which they can use to find the path from source to destination while displaying grievances at particular locations on the map. It helps the user to add markers for source and destination while route is shown using the function called polylines, it can also have response to map gestures. When markers, polygons or other functions are used then an API call is made to the server. With the help of these features additional information can be provided on the map along with user interaction with the map. The API allowsadding these graphics to a map:

\subsubsection{Markers are shown using anchored icons to specific locations}

\subsubsection{Polylines are set of lines used to display the} route

\subsubsection{Polygons are used to show enclosed} segments

4.3.4 Ground Overlays helps to show bitmap graphics at particular positions

\subsubsection{Tile Overlays are displayed using set of} images on top of base map tiles

\subsection{Google Directions API}

The Google Directions API is used with the Google Maps API to show directions on the map with the help of HTTP requests.It helps in searching directions for several modes including walking, cycling and driving. All the data from source to destination is included in this file that is, where to turn, distance between the next turn, locations present on the selected path and which alternative path to select. The polylines helps to draw the path without using any other application to show the required route. Each waypoints, origins and destinations are represented using latitude and longitudes which are used with the polylines to show the particular path. The Directions API also helps to return multipart directions using different waypoints for different alternative routes. The Google Directions helps to calculate the alternatives from source to destination while the data provided in the database is used to compare with each of the co-ordinates in the alternatives to check whether there is high or low traffic and accordingly the correct alternative path is suggested which is going to be the optimized path.

\section{PROPOSED SYSTEM}

This system is an android application which provides the flexibility of using it anywhere at any time. The system takes real time data as input from the users, regarding whether the traffic is low, medium or high and the road of whose traffic he wants to update with the help of GPS information to check whether the mentioned road is available nearby, that is, within a specified radius around his current location. This data is further processed using a scale which is updated according to the condition entered by the user in a timely basis. This data will be further stored in a database along with its date and time.

When users use the application for navigation (Figure 1), it will provide them the optimized path form source to destination from the data stored in the database. When source and destination is provided by the user the co-ordinates are used to provide their intermediate points using Google directions API [7]. These co-ordinates are matched with the database on the server side according to its traffic condition, date and time [10]. This information helps to show the users an optimized route on the map provided by Google maps API.

The system also has a feature of sending alert messages to the concerned authority regarding issues of local governance. For this feature the user sends the description of the issue along with its co-ordinates, which are taken from his GPS and a picture representing the following issue to the application. This grievance is then rated by other users and as the issue becomes severe, it is alerted to the concerned authority. The local governance then uses this information for the maintenance of roads, problems in traffic signals, pot holes, car accidents or any other issue which requires attention. This type of applications can also be used by tourists while travelling in any particular city[11]. 


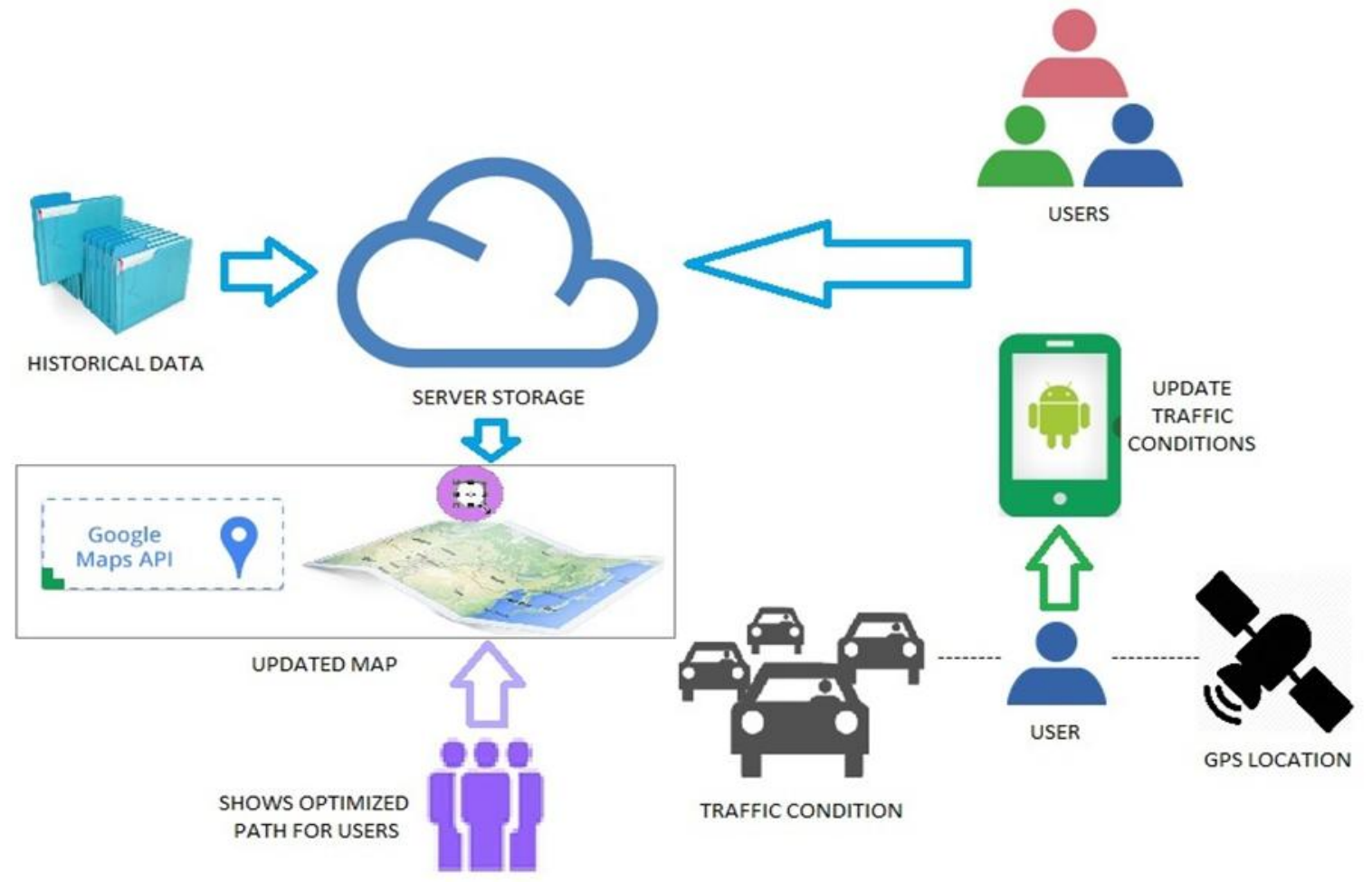

Figure 1: Process of optimized path using people as sensors

\section{ANALYSIS}

The given graph (Figure 2) determines the traffic conditions at particular locations people mention while using the application and how the mentioned conditions are stored in the database. People give the traffic information in a scale from 1-10 evaluated as low (1-3), medium (4-6) and high (710) which is stored in the database. As other people give different traffic condition of the same location, the given values are averaged and the result is stored in the database. It works in a timely basis that is, after each 20 minutes the previous data is discarded and the new traffic conditions are stored since traffic may vary immensely after few minutes and taking average continuously will not be a good idea.

This pattern which immerges from the graph is stored in the database which is used as historical data while determining the traffic conditions for a location at a particular time, if the pattern remains constant for a couple of months. This historical data will help in determining the traffic conditions at a particular day and time like during festivals or when schools and companies start and end. This historical data is directly used even if people mentioned condition is different because sometimes not many people would have actually used the application to mention the traffic condition at that location.

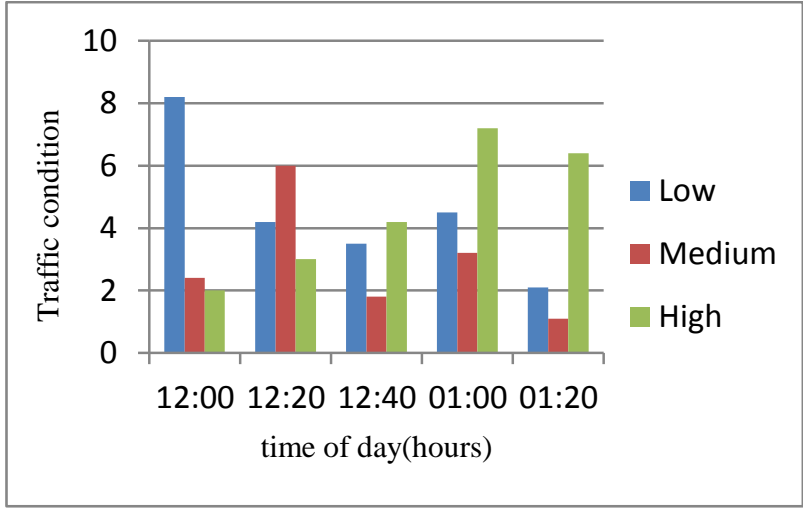

Figure 2: Traffic condition at a particular location and time

\section{CONCLUSION}

In this paper, Google API's along with the database and formulae are used to process real time data available from smartphone users, and provide optimum path that should be taken by the user to reach a specific destination. Traffic patterns drawn over a period of time can help in synchronizing traffic signals in adjacent squares of a city. This eventually can help in bringing a significant change in traffic management. A communication platform provides the people of a city to post their complaints regarding local governance, which if well supported by other users will be notified to the concerned authority.

The same technique can be used for weather detection such as if some area has high weather conditions then the people who want to pass or reach to that particular area can take following precautions. 
This technique can be further used during when people are waiting in a restaurant to get a vacant table can inform other people the time they will need to wait to get a vacant table using the android application. This time will be shown on the map along with the restaurant and the people using the application can select the restaurant which matches their needs without actually visiting the restaurant and waiting in a long line just to get a vacant table.

\section{REFERENCES}

[1] Luis Sánchez , Ignacio Elicegui, Javier Cuesta, Luis Muñoz and Jorge Lanza, "Integration of Utilities Infrastructures in a Future Internet Enabled Smart City Framework",2013 .

[2] P. E. Hart, N. J. Nilsson, and B. Raphael. A Formal Basis for the Heuristic Determination of Minimum Cost Paths. IEEE Transactions on Systems Science and Cybernetics, Vol. SSC-4, No. 2, pages 100-107, 1968.

[3] Liping Cheng, Chuanxi Liu, Bo Yan, "Improved hierarchical A-star algorithm for optimal path planning of the large parking lot", pages 695-698, July 2014.

[4] M. Halvey, T.Keane, and B. Smyth. Predicting Navigation Patterns on the Mobile-Internet Using Time of the Week. In Proceeding of the $14^{\text {th }}$ international conference on World Wide Web, pages 958-959, Chiba, Japan, May 2005.

[5] H. Gonzalez, J. Han, X. Li, M. Myslinska, and J. P. Sondag. Adaptive Fastest Path Computation on a Road Network: A Traffic Mining Approach. In Proceeding of the 14th international conference on Very Large Data Bases, Vienna, Austria, September 2007.
[6] Lu, E.H.-C.; Chia-Ching Lin; Tseng, V.S.,"Mining the Shortest Path within a Travel Time Constraint in Road Network Environments",in proceeding of the $11^{\text {th }}$ International IEEE Conference on Intelligent Transportation Systems, Tainan, October 2008.

[7] Google directions API-Android Developer https://developers.google.com/maps/documentation/direc tions.

[8] BagrechaKomal, S., BramhechaAmit, R., ChhajedSneha, S.andKhivsara, B.A., "Android Application using GPS Navigation", Issue of Internationa 1 Journal of Electronics, Communication and Soft Computing Science and Engineering, Berkeley, vol. 5, pages 84-89, 2012.

[9] A. Prakash and R. Manickavasagam, "Elegant Way of Reaching Destination Using GPS and Drivers Ability", Volume 4, Issue 3, pages 908-911, March 2014.

[10] Xiucheng Yang, Danfeng Liu, Lin Cong, Ligang Liang, "Shortest path algorithm based on distance comparison", pages 3137-3139, July 2014.

[11] ShouChih Lo, Fu Chiand Huang, Chien Chung Wang, "Transport Route Planning for Mobile Tour Applications", pages 387-392, Sept 2014.

[12] Madli, R, Hebbar, S, Pattar, P, GV, P, "Automatic Detection and Notification of Potholes and Humps on Roads to Aid Drivers", Sensors Journal, Volume: PP, Issue: 99, page 1, March 2015. 